\title{
Optimization of fMRI Processing Parameters for Simutaneous Acquisition of EEG/fMRI in Focal Epilepsy
}

\author{
M. Forjaz Secca ${ }^{1,3}$, H.M. Fernandes ${ }^{1}$, J.R. Cabral ${ }^{1}$ and A. Leal ${ }^{3,4}$ \\ ${ }^{1}$ Cefitec, Dep. of Physics, Universidade Nova de Lisboa, Portugal \\ ${ }^{2}$ Ressonância Magnética de Caselas, Lisboa, Portugal \\ ${ }^{3}$ Department of Neurophysiology, Hospital Júlio de Matos, Lisboa, Portugal \\ ${ }^{4}$ Department of Pediatric Neurology, Hospital Dona Estefânia, Lisboa, Portugal
}

\begin{abstract}
In the context of focal epilepsy, the simultaneous combination of electroencephalography (EEG) and functional magnetic resonance imaging (fMRI) holds a great promise as a technique by which the hemodynamic correlates of interictal spikes detected on scalp EEG can be identified.

The fact that traditional EEG recordings have not been able to overcome the difficulty in correlating the ictal clinical symptoms to the onset in particular areas of the lobes, brings the need of mapping with more precision the epileptogenic cortical regions. On the other hand, fMRI suggested localizations more consistent with the ictal clinical manifestations detected.

This study was developed in order to improve the knowledge about the way parameters involved in the physical and mathematical data, produced by the EEG/fMRI technique processing, would influence the final results. The evaluation of the accuracy was made by comparing the BOLD results with: the high resolution EEG maps; the malformative lesions detected in the T1 weighted MR images; and the anatomical localizations of the diagnosed symptomatology of each studied patient. The optimization of the set of parameters used, will provide an important contribution to the diagnosis of epileptogenic focuses, in patients included on an epilepsy surgery evaluation program.

The results obtained allowed us to conclude that: by associating the BOLD effect with interictal spikes, the epileptogenic areas are mapped to localizations different from those obtained by the EEG maps representing the electrical potential distribution across the scalp (EEG); there is an important and solid bond between the variation of particular parameters (manipulated during the fMRI data processing) and the optimization of the final results, from which smoothing, deleted volumes, HRF (used to convolve with the activation design), and the shape of the Gamma function can be certainly emphasized.
\end{abstract}

Keywords - fMRI, BOLD, EEG, Epilepsy.

\section{INTRODUCTION}

The simultaneous acquisition of electroencephalography (EEG) and functional magnetic resonance imaging (fMRI) has been increasingly used in focal epilepsy to correlate the hemodynamic response to the interictal spikes detected on EEG. However EEG recordings have presented some difficulties in correlating the ictal clinical symptoms to the onset in particular areas of the lobes, whereas fMRI suggested localizations more consistent with the ictal manifestations.

To further understand the differences between EEG and fMRI localizations it is important to study how the different parameters involved in the processing of physical and mathematical fMRI data can influence the final results in terms of consistency and proximity to the lesions observed.

In order to achieve that we analyzed two of our patients in detail, by varying some of the main fMRI processing parameters and compared the results with the EEG data and the lesion locations.

\section{MATERIALS AND METHODS}

We studied two patients with a diagnosis of idiopathic OLE, submitting them to a 120-min EEG recording outside the scanner, with a cap of $64 \mathrm{AgCl}$ electrodes. The position of each electrode was obtained using an electromagnetic digitizer PATRIOT. We used a sampling rate of $256 \mathrm{~Hz}$, filters of $0.5-70 \mathrm{~Hz}$. One week after this procedure, a session of functional MRI was performed while simultaneously recording the EEG (37 eletrvodes along the scalp and 2 located in the chest). Each patient demonstrated a single, topographically stable, paroxysm type. This was the main neurophysiological criteria for selection of patients for this study. Informed consent was obtained from the parents of the patients.

Source analysis of the EEG was done in spikes detected visually in recordings obtained outside the scanner for both patients. The EEG was high pass filtered at $3 \mathrm{~Hz}$, and spikes with good signal to noise ratio were aligned by the peak amplitude to produce an average spike. The sources were obtained from instantaneous regional dipoles at the peak of averaged spikes, with a standard three layer Boundary Element Model (BEM) of volume conduction (conductances of $0.33,0.0042,0.33 \mathrm{~S} / \mathrm{m}$ for scalp, bone and brain), provided in the Source2 software package (Neuroscan, El Paso, Mexico). Standard electrode positions were also used.

The EEG/fMRI consisted in the acquisition of blocks of 150 brain volumes for patient 1 and 140 brain volumes for patient 2, each one made of 34 EPI images (in plane resolu- 
tion $3.75 \mathrm{~mm}$ and slice thickness of $7 \mathrm{~mm}$, no spacing; FOV: $24 \times 24 \mathrm{~cm}$; matrix: $64 \times 64$; flip angle of $90^{\circ}$ ) obtained with a $\mathrm{TE}=50 \mathrm{~ms}, \mathrm{a} \mathrm{TR}=2.275 \mathrm{~s}$ (patient 1 ) and TR $=2.475 \mathrm{~s}$, (patient 2), corresponding to periods of $351 \mathrm{~s}$ and $357 \mathrm{~s}$ of continuous and simultaneous monitoring. 5 blocks were obtained per patient, providing $175 \mathrm{v} 5 \mathrm{~s}$ of simultaneous monitoring for patient 1 , and $1785 \mathrm{~s}$ for patient 2 . A brain $\mathrm{T} 1$ weighted anatomic sequence (in plane resolution $0.94 \mathrm{~mm}$ and slice thickness of $1.3 \mathrm{~mm}$ ) was obtained in the same session.

Images were acquired in a $1.5 \mathrm{~T} \mathrm{GE} \mathrm{CVi} / \mathrm{NVi}$ scanner, while the EEG was recorded through a set of $\mathrm{AgCl}$ electrodes connected to an amplifier located outside the scanner room through carbon fiber wires (MagLink, Neuroscan, El Paso, TX, U.S.A.). The cap did not produce detectable artifacts in the MRI sequences, so these could be processed without any special correction. The EEG was corrected for artifacts induced by the magnetic field and rapidly changing imaging gradients offline using commercial software (Scan

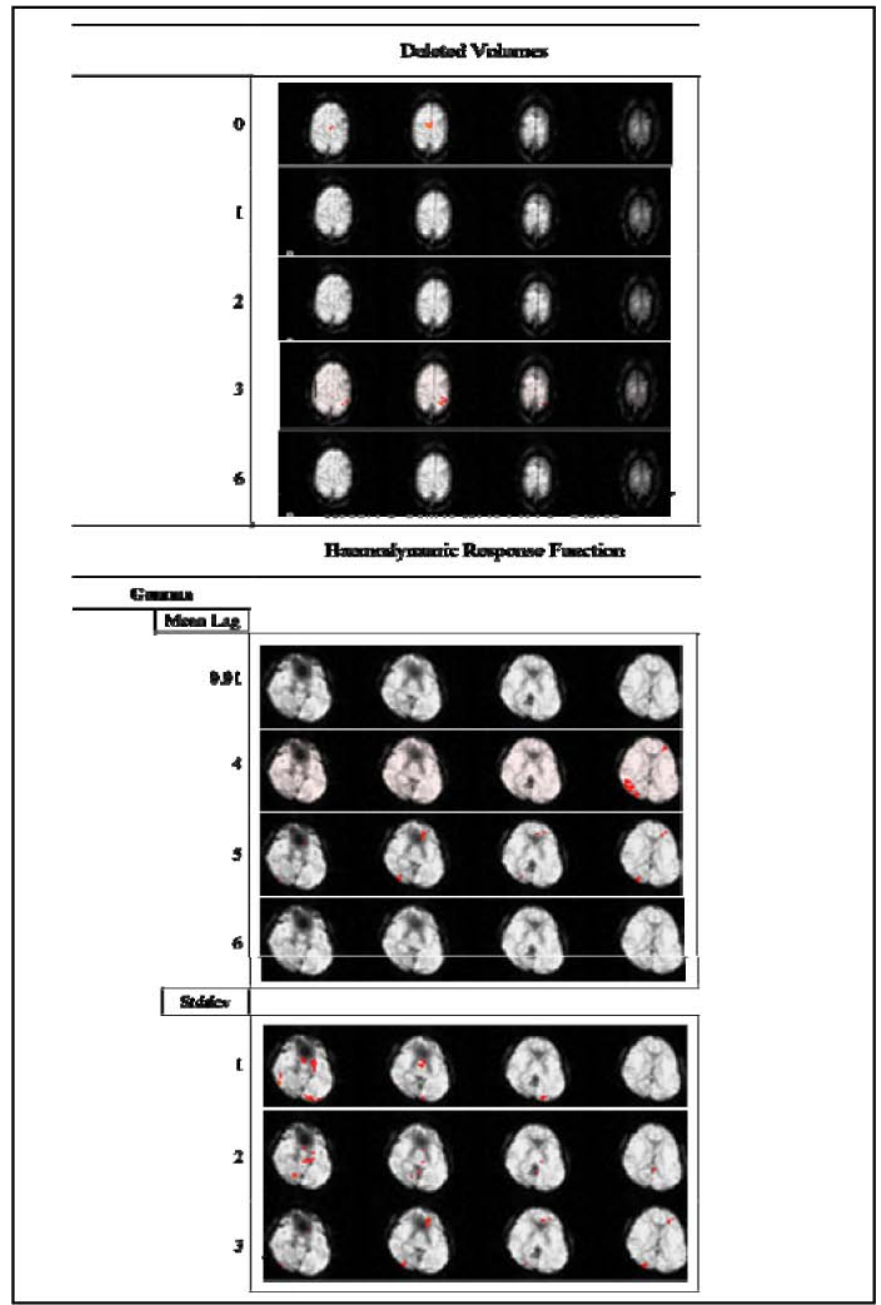

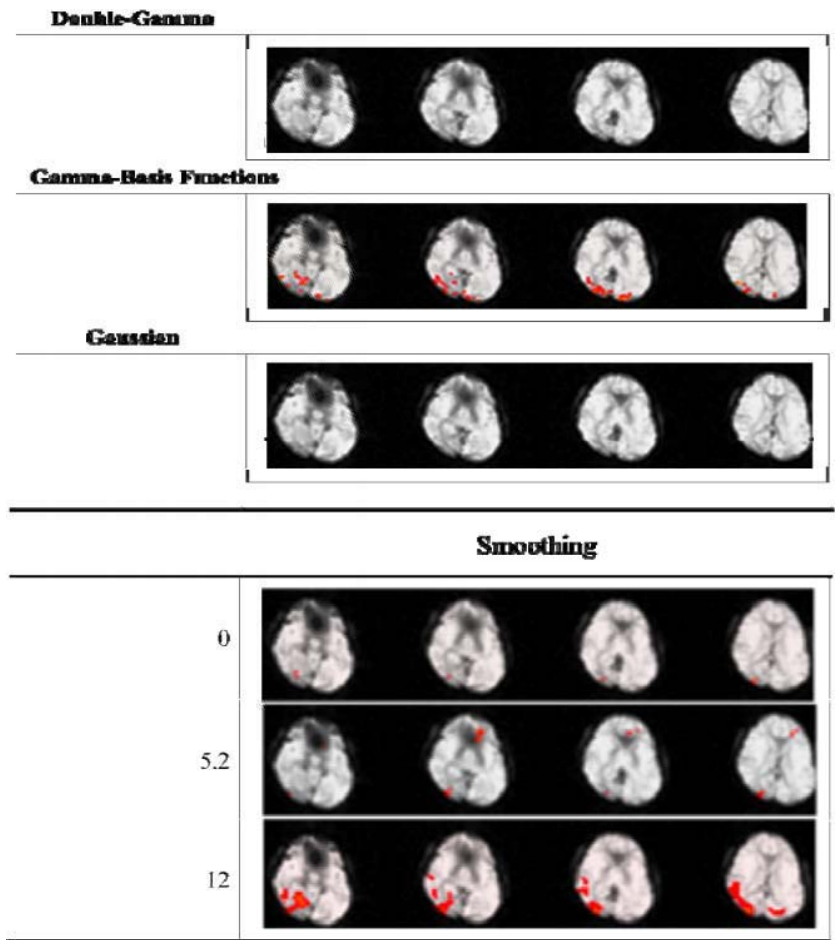

Fig. 1 Variation of some important parameters used in the fMRI data preprocessing. The left side of each brain slice image corresponds to the right side of the real brain.

4.3.2, Neuroscan). The time of occurrence of spikes was determined by visual inspection and used to classify the acquired image volumes, resulting in sequences of events of interest used to build an event-related paradigm.

The preprocessing and paradigm-related analysis of the fMRI was performed using the FSL software package [1]. In order to perform this study, a set of important parameters, involved in the fMRI data preprocessing, was initially selected. The tests presented along the study reveal the influence of each parameter in the accuracy of BOLD activation maps, and also its contribution to false positives. To model the haemodynamic function a standard Gamma function with derivatives was used [2] (with a FWHM of $3 \mathrm{~s}$ [3] and a mean lag of $5 \mathrm{~s}$ [4]). Four and twenty-four spikes were analyzed, respectively, for patients 1 and 2. The EPI sequences were corrected for movement and slice acquisition time and smoothed with a Gaussian kernel of FWHM $5.2 \mathrm{~mm}$ [5]. No initial volumes from the acquired fMRI sequence were deleted. A local autocorrelation correction was used and $\mathrm{z}$ statistic images generated. The correction for the multiple comparison problem was done using a cluster threshold with $\mathrm{p}=0.05$. [3]

Representation of dipoles on individual brain anatomy was performed by adjusting the fiducial points (nasion, 
preauricular points, inion, and vertex) of the BEM model on the individual 3D T1 MRI.

\section{RESULTS}

In order to improve the knowledge about how parameters involved in the fMRI data preprocessing influence the final results, we have performed several tests consisting in varying each parameter in a certain range, which included the group of values that had already been verified as scientifically reasonable.

The results of each test are represented by a sequence of 4 axial slices of the brain that includes the region of interest for each patient. The identification of this region was made by combining both information of the malformative lesion detected in the T1 weighted MR image, and the anatomical localization of the diagnosed symptomatology of each studied patient.

We have observed that each patient is a case on its own and should be studied individually. In this paper, in order to demonstrate the importance of these parameters and their individual variation we present the results of two of our patients.

For the "Deleted Volumes" test (patient 2), different BOLD activation maps were performed. The option of deleting the first 3 volumes of this sequence shows to be the best option, since the BOLD clusters detected are over the primary motor cortex area (Fig. 2, 3b), considerably near from the malformative lesion (Fig. 3a). These results are also consistent with the diagnosed symptomatology, since the patient has moved his right hand during the seizures.

In the study for the "Haemodynamic Response Function" (patient 1) parameter were included another two tests, in order to evaluate the influence of the Gamma function's shape in the BOLD activation distribution map. The "Mean Lag" test showed that there was a restricted range of values (4 and 5) where it was possible to identify some BOLD clusters. The "Stddev" test for $1 \mathrm{~s}$ reveals a cluster located over the cortical left occipital region, in the same axial plan as the malformative lesion detected for this patient. For the rest of the haemodynamic response functions studied, only the set of Gamma-basis functions did show some BOLD clusters. Some of these clusters seem to be highly significant since they are near the expected area (Fig. 1, 2b).

The test for "Smoothing" (patient 2) reveals the great influence of this parameter in the production of accurate BOLD activation maps, since it is intimately related to the size of the clusters. However, its bad use may hide important clusters or even produce false positives.

The evaluation of the accuracy of the results that showed better correlation to the expected information, was made by
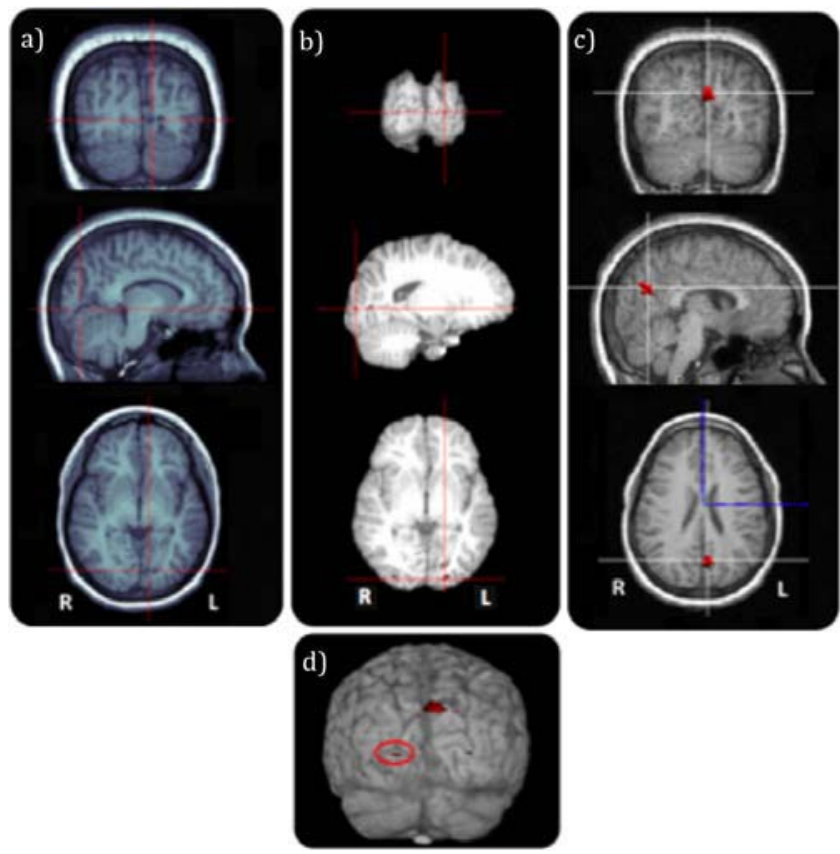

Fig. 2 Patient 1: (a) T1 weighted MR images identifying the malformative region; (b) BOLD fMRI images showing the cluster over the cortical left occipital region, near the malformative region; (c) Regional dipole model applied to calculate the electric source of the detected spike (EEG). (d) 3D fMRI BOLD activation map (rendering).

Table 1 Coordinates corresponding to the center of the detected malformative lesion, cluster BOLD and EEG source position for patient 1.

\begin{tabular}{lccc}
\cline { 2 - 4 } & \multicolumn{3}{c}{ Position } \\
\hline X (mm) & Y (mm) & $\mathrm{Z}(\mathrm{mm})$ \\
\hline Lesion & 128 & 62 & 64 \\
Cluster BOLD & 138 & 51 & 61 \\
Regional Dipole (EEG)* & 126 & 71 & 85 \\
\hline
\end{tabular}

Table 2 Distances between the cluster BOLD, the lesion, and the regional dipole for patient 1 .

\begin{tabular}{lcc}
\cline { 2 - 3 } & Lesion $(\mathrm{mm})$ & Regional Dipole $(\mathrm{mm})$ \\
\hline Cluster BOLD & 15.17 & 33.47 \\
Regional Dipole & 22.93 & - \\
\hline
\end{tabular}

Table 3 Coordinates corresponding to the center of the detected malformative lesion, cluster BOLD and EEG source position for patient 1 .

\begin{tabular}{lccc}
\cline { 2 - 4 } & \multicolumn{3}{c}{ Position } \\
\hline & $\mathrm{X}(\mathrm{mm})$ & $\mathrm{Y}(\mathrm{mm})$ & $\mathrm{Z}(\mathrm{mm})$ \\
\hline Lesion & 140 & 89 & 104 \\
Cluster BOLD & 143 & 92 & 101 \\
Regional Dipole (EEG)* & 150 & 109 & 98 \\
\hline
\end{tabular}


Table 4 Distances between the 3 types of results for patient 2 .

\begin{tabular}{lcc}
\cline { 2 - 3 } & Lesion & Regional Dipole \\
\hline Cluster & 5.20 & 19.32 \\
Regional Dipole & 23.15 & - \\
\hline
\end{tabular}
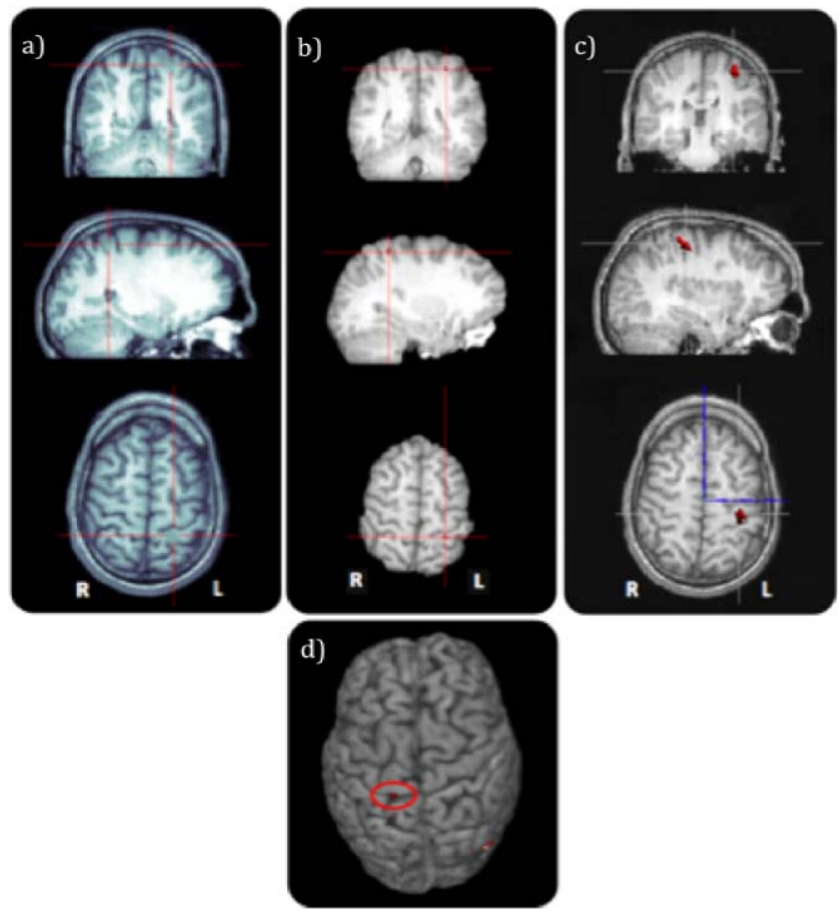

Fig.3. Patient 2: (a) T1 weighted MR images identifying the malformative region; (b) BOLD fMRI images showing the cluster over the primary motor cortex area, near the malformative region; (c) Regional dipole model applied to calculate the electric source of the detected spike (EEG). (d) 3D fMRI BOLD activation map (rendering).

comparing the BOLD results with: the Regional Dipole EEG maps (Fig. 2c, 3c); the malformative lesions detected (Fig. 2a, 3a) in the T1 weighted MR images; and the anatomical localizations of the diagnosed symptomatology of each studied patient. The correlation between the results (for the best set of parameters) has been analyzed through the measurement of the distances between the center of each focus for patient 1 and 2 (Table 2,4).

The results reflect a very good correlation between the malformative lesion and the cluster BOLD (closest to the expected area) for each patient. For The Regional Dipole results presented a reliable GOF $(0.959$ and 0.953 for patient 1 and 2), and the estimated EEG sources are about 2.3 $\mathrm{cm}$ apart from the malformative lesion for both patients, showing also a good correlation between results.

\section{Conclusions}

The results obtained allowed us to conclude that: by associating the BOLD effect with interictal spikes, the epileptogenic areas are mapped to localizations different from those obtained by regional dipole maps (EEG); there is an important and solid bond between the variation of particular parameters (manipulated during the fMRI data processing) and the optimization of the final results, from which smoothing, deleted volumes, HRF (used to convolve with the activation design), and the shape of the Gamma function can be certainly emphasized.

\section{AcKNOwLedGMENT}

The work was supported by a grant for Research in Epilepsy from Tecnifar SA and by projects Topo3D (POSI/CPS/39758/2001) and EpilBI (POSC/EEACPS/60977/2004) from FCT.

\section{REFERENCES}

1. Smith S, Jenkinson M, Woolrich M, et al. Advances in functional and structural MR image analysis and implementation as FSL. Neuroimage 2004;23(suppl 1):208-19.

2. Huettel S, McKeown J, Song A, et al. Linking hemodynamic and electrophysiological measures of brain activity: evidence from functional MRI and intracranial field potentials. Cereb Cortex 2004;4:165-73.

3. Analysis Group, FMRIB, Oxford, UK. FSL. FEAT - FMRI Expert Analysis Tool. [Online] [Citação: 7 de Apr de 2007.] http://fsl.fmrib.ox.ac.uk/fsl/feat5/.IFMBE at http://www.ifmbe.org .

4. Kang, Joong Koo, et al. Using patient-specific hemodynamic response functions in combined. NeuroImage. 2003, Vol. Oct, 11621170.

5. Hawco, Colin S, et al. BOLD changes occur prior to epileptic spikes seen on scalp EEG. NeuroImage. May 1, 2007, Vol. 35(4), 14501458.

Author: Mario Forjaz Secca

Institute: Cefitec, Physics Department, Universidade Nova de Lisboa

Street: Quinta da Torre

City: 2829-516 Caparica

Country: Portugal

Email: mfs@fct.unl.pt 